\title{
Molecular Dynamics Characteristics and Model of Vehicle-Following Behavior
}

\author{
Yanfeng Jia $\mathbb{D}^{1},{ }^{1}$ Dayi Qu $\left(\mathbb{D},{ }^{1}\right.$ Xiaolong Ma $\mathbb{D}^{2},{ }^{2}$ Lu Lin $\mathbb{D}^{1},{ }^{1}$ and Jiale Hong $\mathbb{D}^{1}$ \\ ${ }^{1}$ School of Mechanical and Automotive Engineering, Qingdao University of Technology, Qingdao 266520, China \\ ${ }^{2}$ National ITS Center of Engineering and Technology, Qingdao 266500, China \\ Correspondence should be addressed to Yanfeng Jia; 771210072@qq.com and Dayi Qu; dyqu@263.net
}

Received 14 July 2020; Revised 5 October 2020; Accepted 28 November 2020; Published 7 December 2020

Academic Editor: Shamsunnahar Yasmin

Copyright (c) 2020 Yanfeng Jia et al. This is an open access article distributed under the Creative Commons Attribution License, which permits unrestricted use, distribution, and reproduction in any medium, provided the original work is properly cited.

\begin{abstract}
The vehicle-following behavior is a self-organizing behavior that restores dynamic balance under the stimulation of external environmental factors. In fact, there are asymmetric problems in the process of acceleration and deceleration of drivers. The existing traditional models ignored the differences between acceleration and deceleration of vehicles. In order to solve this problem, the vehicles driving on the road are compared to interacting molecules. Vehicle-following characteristics are studied, and the molecular following model is established based on molecular dynamics. The model parameters under different conditions are calibrated considering the required safety distance by the vehicle and the reaction time of the driver. With the help of the vehicle running track graphs, speed, and acceleration graphs, the numerical simulations of the molecular following model and the classical optimal speed vehicle-following model are carried out. The results of the comparative analysis show that the acceleration in the process of acceleration and deceleration is not constant but more sensitive to the deceleration of the preceding vehicle than to the acceleration and more sensitive to the acceleration/deceleration of the short-distance vehicle than to the acceleration/deceleration of the long-distance vehicle. Therefore, the molecular following model can better describe the vehicle-following behavior, and the research results can provide a theoretical basis and a technical reference for the analysis of traffic flow dynamic characteristics and adaptive cruise control technology.
\end{abstract}

\section{Introduction}

The vehicle-following behavior is an important part of traffic safety. The concept of vehicle-following (CF) originated in the early 1950s and was proposed by Pipes [1]. Scholars have conducted systematic research on the vehicle-following model. After more than 60 years of development, the related research on the vehicle-following model has also been continuously improved and perfected, so that a variety of situations have emerged. According to their starting points and angles, they can be divided into two categories: datadriven and theoretical-driven.

Data-driven model: by extracting and calculating the measured data, a large number of high-precision and highquality vehicle trajectory data are fitted to establish a vehiclefollowing model. Although this type of model does not have a very clear physical meaning, it can reasonably display and predict complex traffic phenomena that cannot be simulated by some data-driven vehicle-following models. Among them, the most widely used is the backpropagation (BP) neural network proposed by Rumelhart et al. [2]. Subsequently, Kehtarnavaz et al. first applied BP neural network in traffic simulation to model the vehicle-following behavior in [3]. By entering the speed of the following vehicle and the headway between the vehicle pair, the relative speed of the two vehicles can be obtained. Later, other scholars began to study the vehicle-following behavior based on neural networks [4-6], but the BP neural network model requires a lot of data for training. During the training process, the training will produce a large error prediction for extreme values (such as acceleration equal to zero), and because this type of model does not introduce the relevant theory of vehicle following, its simulation of some vehicle-following behaviors may even have errors $[7,8]$. Therefore, support vector 
regression was subsequently used to study vehicle following. In 2010, Wei first proposed a vehicle-following model based on support vector regression in [9]. Its input is the speed of the following vehicle, the relative speed of the two vehicles, and the vehicle distance at the current moment, and the output is the speed of the following vehicle at the next moment. The evaluation index shows that the model has obtained good simulation results. Later, with the emergence of online traffic simulation technology, Wang established a vehicle-following model based on online support vector regression in [10]. Theoretically, the use of support vector regression to model the vehicle-following behavior is better, but the current research based on such models is still in its infancy, and there are not many relevant results.

Theory-driven model: according to fuzzy theory, chaos theory, nonchaos theory, and other related theories of vehicle-following behavior, the vehicle-following models were established by using differential and dynamic knowledge. The physical meaning of this type of model is clear, and it is used in many kinds of traffic simulation software. In the development of theory-driven vehicle-following models, the stimulus-response model was the most classic, including the famous General Motors (GM) model [11]. It can be said that many stimulus-response models are mostly based on the GM model. The GM model assumes that the vehicle does not overtake or change lanes while following the vehicle. Derived from the driving dynamics theory, the basic formula is

$$
a_{n+1}(t+T)=\lambda v_{n+1}^{m}(t+T) \frac{\Delta v(t)}{[\Delta x(t)]^{l}},
$$

where $a_{n+1}(t+T)$ is the instantaneous acceleration of the following vehicle at time $(t+T) ; v_{n+1}(t+T)$ is the instantaneous velocity of the following vehicle at time $(t+T)$; $\Delta v(t)$ is the speed difference between the vehicle pair at time $t$; Error Reference source not found is the space headway between the vehicle pair at time $t$; $T$ is the reaction time; $\lambda$ is the sensitivity parameter; $m$ and $l$ are also parameters to be calibrated.

It can be seen that the GM model has the characteristics of simple form, clear physical meaning of each part, and groundbreaking, and it can better reflect the vehicle-following characteristics, but there is a problem that the vehicle has excessive acceleration during parking and starting in this model. Later, Newell proposed a new vehicle-following model in [12], which constantly adjusts the speed of the following vehicle to achieve the optimal speed according to the changing distance between two vehicles. This also laid the foundation for the later optimal velocity (OV) model. The OV model is now widely used. It can reasonably describe the state change of macrotraffic flow from the microscopic simulation, but the model also has some shortcomings; that is, unrealistic traffic phenomena will occur during the simulation; for example, the acceleration value of some vehicles may be too high, the speed data may appear negative value, and the vehicle may collide. To deal with these problems, many scholars have continued to improve and optimize them, so that the model continued to improve [13-18].
In summary, whether it is a classic GM model or a subsequent OV model, the interpretation of the macroscopic phenomenon of traffic flow is more in line with the actual situation. But the accuracy of the microinteraction between vehicles varies greatly, and they ignore the asymmetry in the acceleration and deceleration operations caused by the driver's psychological changes and vehicle performance.

Aiming at the problems of the theory-driven model, this paper follows the physical law that "force is the internal cause of changing the motion state of the object," starting from the single-lane vehicle interaction relationship, using the molecular interaction relationship to describe the vehicle-following behavior. Like the traffic on the road to particles, the change of individual's motion state may cause the corresponding change of other individuals' motion state. This dynamic associated change is an interactive behavior, which can be regarded as a coupling relationship. The distance between vehicles traveling on the road is neither too far nor too close, which is very similar to the phenomenon of molecular motion. Figure 1 shows the relationship between the forces of the molecules, where $r=r_{0}$ is the equilibrium distance between molecules.

As shown in Figure 2, there is also a dynamic following balance distance between the vehicles at different speeds, which is called the required safe distance. It is a flexible mapping for following vehicles to drive safely. The change in the direction of the resultant force on following vehicle drives the vehicle to accelerate or decelerate. When $X=L$, following vehicle and leading vehicle just keep the required safe distance and are in a steady-state balance. When $X<L$, following vehicle is accelerated by gravitation; when $X>L$, following vehicle is decelerated by repulsive force. The vehicle-following behavior is the operation of searching for a suitable safety distance and then accelerating or decelerating. From a physical point of view, the change of the vehicle's state is the movement under the combined action of the gravitation and repulsion of the surrounding vehicles, which is similar to the behavior between molecules. This physical phenomenon between molecules provides inspiration for this paper.

The following content of this paper is divided into four parts: the second section is based on dynamics to establish a single-lane molecular following model; the third section is to use the NGSIM data set and the driver's reaction time to calibrate the parameters of the molecular vehicle-following model; the fourth section is the numerical simulation of the built model and the classic OV follow-up model; the fifth section is the analysis and discussion of the simulation results; the sixth section is a summary and prospect of the research results achieved in this paper.

\section{Methods}

2.1. Single-Lane Molecular Vehicle-Following Model. The single-lane vehicle following can be regarded as a linear motion model on only one of the coordinate axes in twodimensional space. According to the change characteristics of the single-lane vehicle following, it is divided into 4 


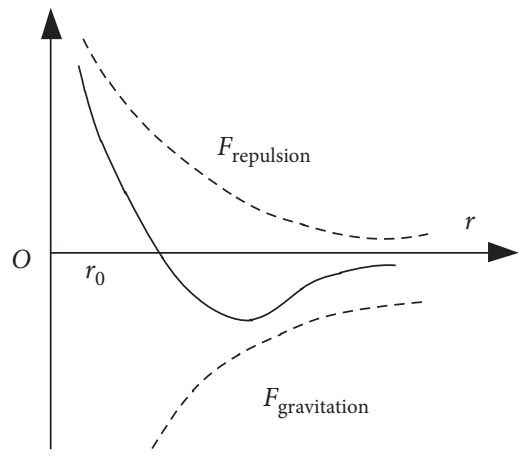

Figure 1: Molecular forces.

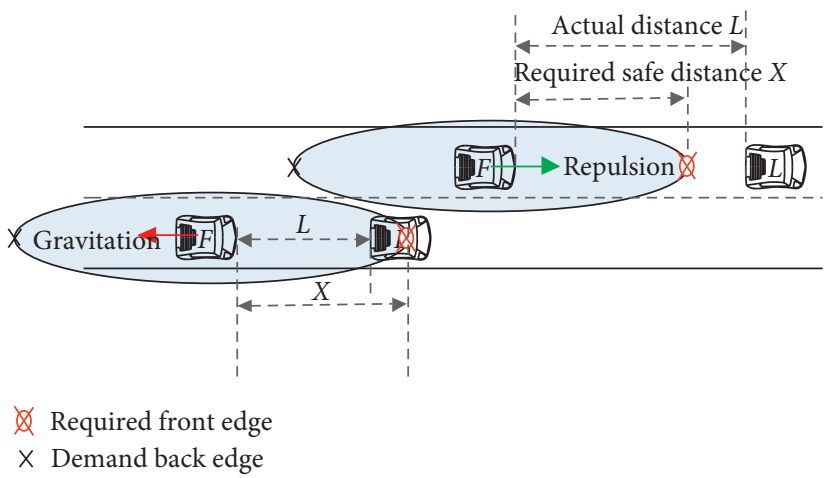

Figure 2: Force diagram of vehicle following.

processes, as shown in Figure 3. They are original following, start of reaction, variable speed stage, and secondary balance:

State 0 is the original following; that is, the distance between the two vehicles is kept to drive stably at the required safe distance. At this time, satisfy $L_{0}=X_{n}=X_{L 0}-X_{F 0}$.

State $0 \longrightarrow$ State 1 : the leading vehicle starts to perform a uniform deceleration motion as the acceleration $a_{L}$ and the duration is $t_{1}$. The distance between the two vehicles is gradually smaller than the required safe distance; that is, $X_{L 1}-X_{F 1}=L_{1}<X_{n}$; this stage is also the reaction time of the following driver.

State $1 \longrightarrow$ State 2: the leading vehicle continues to do a uniform deceleration with acceleration $a_{L}$ and the duration is $t_{2}$, and when it reaches State 2, it will start driving at a constant speed. The following vehicle starts to decelerate at an average acceleration $a_{F}$.

State $2 \longrightarrow$ State 3: the leading vehicle keeps moving at a constant speed, and the following vehicle also recovers its constant speed when it reaches State 3 after $t_{3}$ time. After that, the following vehicle achieves secondary balance.

The equation can be obtained from State $0 \longrightarrow$ State 2 :

$$
\left\{\begin{array}{l}
L_{0}+d_{3}=L_{2}+d_{2}, \\
d_{3}=t_{1} \cdot v_{L 0}+t_{1}^{2} \cdot \frac{a_{L}}{2}, \\
d_{2}=t_{1} \cdot v_{F 0}+t_{2} v_{F 0}+t_{2}^{2} \cdot \frac{a_{F}}{2},
\end{array}\right.
$$

where $v_{F 0}, v_{L 0}$ are the initial speeds of following vehicle and leading vehicle, respectively, and $v_{F 0}=v_{L 0} ; d_{2}, d_{3}$ are the distance traveled by following vehicle and leading vehicle at this stage, respectively.

The equation also can be obtained from State $2 \longrightarrow$ State 3:

$$
\left\{\begin{array}{l}
L_{2}+d_{5}=L_{3}+d_{4}, \\
d_{5}=t_{3} \cdot v_{L 1}, \\
d_{4}=t_{3} \cdot v_{F 1}+t_{3}^{2} \cdot \frac{a_{F}}{2},
\end{array}\right.
$$

where $v_{F 1}, v_{L 1}$ are the speeds of following vehicle and leading vehicle when they reach State 2; $v_{L 1}$ can be calculated from $v_{L 1}=v_{L 0}-a_{L} \cdot t_{2} ; \quad v_{F 1} \quad$ can be calculated from 


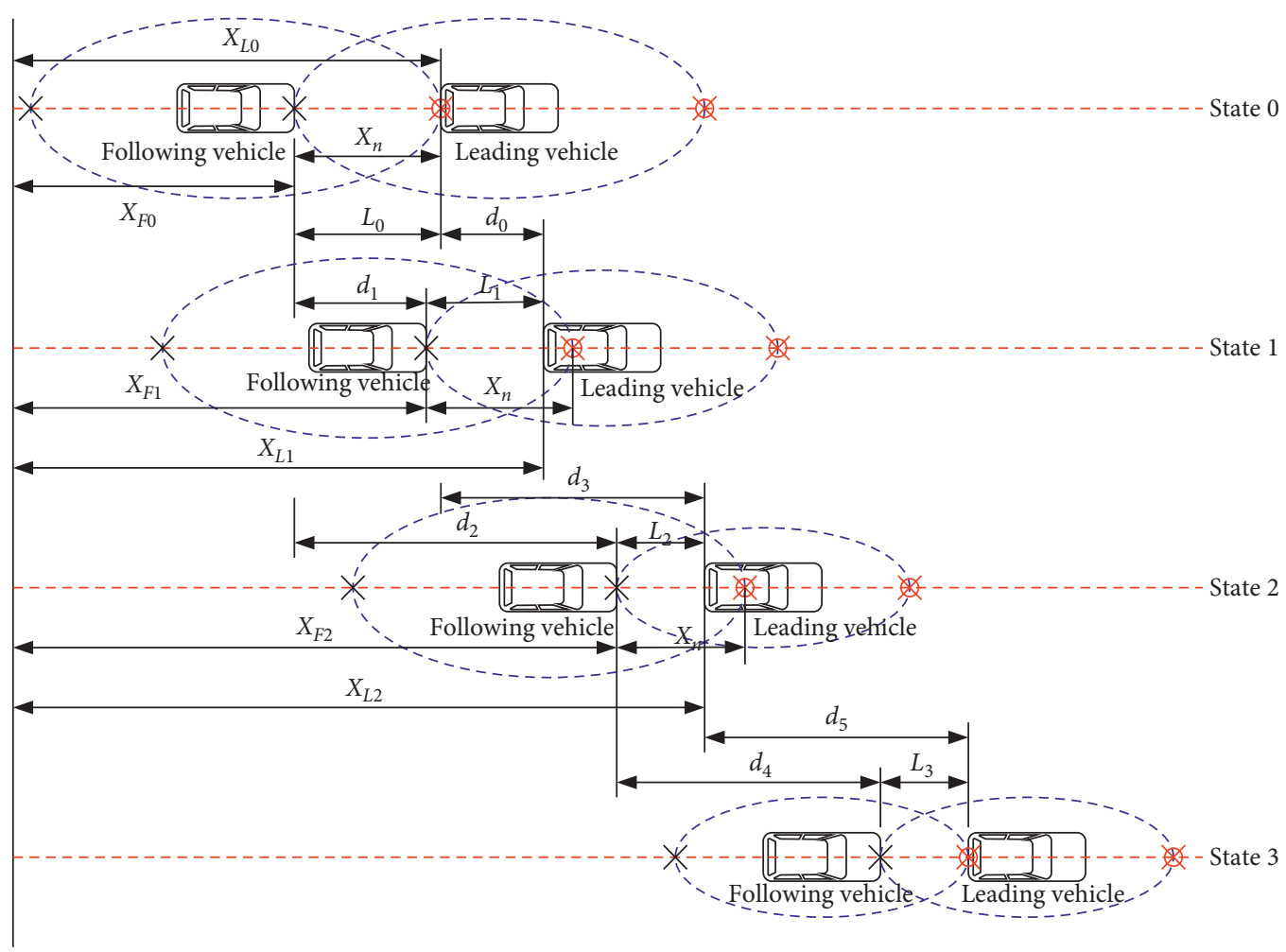

FIGURE 3: Analytical diagram of speed change of single-lane-following model.

$v_{F 1}=v_{F 0}-a_{F} \cdot t_{2} ; d_{5}, d_{4}$ are the distances traveled by the front and rear vehicles during time $t_{3}$, respectively.

By combining equations (2) and (3), we can get

$$
a_{F}=\frac{2}{t_{2}^{2}}\left[\frac{2 t_{2} t_{3}-t_{3}^{2}}{t_{1}^{2}}\left(L_{2}-X_{n}\right)+t_{2}\left(v_{L 1}-v_{F 1}\right)+\frac{t_{1}^{2}}{t_{3}^{2}}\left(L_{2}-L_{3}\right)\right] \text {. }
$$

In order to better describe the influencing factors of the following vehicle's acceleration, let $t_{2}=b t_{1}, t_{3}=c t_{1}$ and simplify equations (4) to (5):

$$
a_{F}=\frac{2}{b^{2} t_{1}^{2}}\left[\left(2 b c-c^{2}\right)\left(L_{2}-X_{n}\right)+b t_{1}\left(v_{L 1}-v_{F 1}\right)+\frac{1}{c^{2}}\left(L_{2}-L_{3}\right)\right] \text {. }
$$

Introducing dimensionless $\alpha, \beta$, and $\gamma$ to make

$$
\begin{aligned}
& \frac{2}{b^{2} t_{1}^{2}}\left[\alpha\left(L_{2}-X_{n}\right)+\beta t_{1}\left(v_{L 1}-v_{F 1}\right)+\gamma\left(L_{2}-L_{3}\right)\right] \\
& =\frac{2}{b^{2} t_{1}^{2}}\left[\left(2 b c-c^{2}\right)\left(L_{2}-X_{n}\right)+b t_{1}\left(v_{L 1}-v_{F 1}\right)+\frac{1}{c^{2}}\left(L_{2}-L_{3}\right)\right] .
\end{aligned}
$$

Set $L_{2}-X_{n}=k\left(L_{3}-L_{2}\right), v_{L 1}=p \cdot v_{F 1}$; for deceleration, set $t_{1} \cdot v_{F 1}=q\left(L_{2}-L_{3}\right)$; for acceleration, set $t_{1} \times v_{F 1}=q\left(L_{3}-L_{2}\right)$; and for $k>0, p, q<1$, substitute them into equation (6) and we can get

$$
\begin{aligned}
& {[\alpha k+\beta q(1-p)+\gamma]\left(L_{2}-L_{3}\right) }=\left[k\left(2 b c-c^{2}\right)+b q(1-p)-1\right] \\
& \cdot\left(L_{2}-L_{3}\right) .
\end{aligned}
$$

When only considering the influence of $\left(L_{2}-X_{n}\right)$, then $\gamma \longrightarrow 0$ and $\beta \longrightarrow 0$; we can obtain

$$
\alpha k=k\left(2 b c-c^{2}\right)+b q(1-p)-1 .
$$

Then $\quad\left(2 \alpha / b^{2} t_{1}^{2}\right)=\left(2 / b^{2} t_{1}^{2}\right)\left[\left(k\left(2 b c-c^{2}\right)+b q(1-p)-\right.\right.$ $1 / k)]$ is the driver's response intensity to $\left(L_{2}-X_{n}\right)$.

When only considering the influence of $\left(v_{L 1}-v_{F 1}\right)$, then $\alpha \longrightarrow 0$ and $\gamma \longrightarrow 0$; we can obtain

$$
\beta q(1-p)=k\left(2 b c-c^{2}\right)+b q(1-p)-1
$$

Then $\quad\left(2 \beta / b^{2} t_{1}^{2}\right)=\left(2 / b^{2} t_{1}^{2}\right)\left[\left(k\left(2 b c-c^{2}\right)+b q(1-p)\right.\right.$ $-1 / q(1-p))]$ is the driver's response intensity to $\left(v_{L 1}-v_{F 1}\right)$.

When only considering the influence of $\left(L_{2}-L_{3}\right)$, then $\alpha \longrightarrow 0$ and $\beta \longrightarrow 0$; we can obtain

$$
\gamma=k\left(2 b c-c^{2}\right)+b q(1-p)-1 .
$$

Then $\left(2 \gamma / b^{2} t_{1}^{2}\right)=\left(2 / b^{2} t_{1}^{2}\right)\left[k\left(2 b c-c^{2}\right)+b q(1-p)-1\right]$ is the driver's response intensity to $\left(L_{2}-L_{3}\right)$.

Set $\quad m \geq 1, n \geq 1, h \geq 1$, and $(1 / m)+(1 / n)+(1 / h)=1$. Let $(1 / m),(1 / n)$, and $(1 / h)$ be the respective weights of $\left(L_{2}-X_{n}\right),\left(v_{L 1}-v_{F 1}\right)$, and $\left(L_{2}-L_{3}\right)$ in the factors that affect the acceleration of the following vehicle, multiply them by 
the reaction intensity under each influencing factor, and add up them; we can obtain

$$
\frac{\alpha}{m} k+\frac{\beta}{n} q(1-p)+\frac{\gamma}{h}=k\left(2 b c-c^{2}\right)+b q(1-p)-1 .
$$

By combining equation (5), the acceleration equation under deceleration can be obtained

$$
\begin{aligned}
a_{F}= & \frac{2\left[k\left(2 b c-c^{2}\right)+b q(1-p)-1\right]}{m k b^{2} t_{1}^{2}}\left(L_{2}-L_{n}\right) \\
& +\frac{2\left[k\left(2 b c-c^{2}\right)+b q(1-p)-1\right]}{n q(1-p) b^{2} t_{1}}\left(v_{L 1}-v_{F 1}\right) \\
& +\frac{2\left[k\left(2 b c-c^{2}\right)+b q(1-p)-1\right]}{h b^{2} t_{1}^{2}}\left(L_{2}-L_{3}\right) .
\end{aligned}
$$

Transform equation (11) into

$$
\left\{\begin{array}{l}
a_{F}=\lambda_{0}\left(L_{2}-X_{n}\right)+\lambda_{1}\left(v_{L 1}-v_{F 1}\right)+\lambda_{2}\left(L_{2}-L_{3}\right) \\
\lambda_{0}=\frac{2\left[k\left(2 b c-c^{2}\right)+b q(1-p)-1\right]}{m k b^{2} t_{1}^{2}} \\
\lambda_{1}=\frac{2\left[k\left(2 b c-c^{2}\right)+b q(1-p)-1\right]}{n q(1-p) b^{2} t_{1}} \\
\lambda_{2}=\frac{2\left[k\left(2 b c-c^{2}\right)+b q(1-p)-1\right]}{h b^{2} t_{1}^{2}}
\end{array}\right.
$$

Similarly, the acceleration equation in the accelerated state can be obtained:

$$
\left\{\begin{array}{l}
a_{F}=\lambda_{0}\left(L_{2}-X_{n}\right)+\lambda_{1}\left(v_{L 1}-v_{F 1}\right)+\lambda_{2}\left(L_{2}-L_{3}\right), \\
\lambda_{0}=\frac{2\left[k\left(2 b c-c^{2}\right)+b q(p-1)-1\right]}{m k b^{2} t_{1}^{2}} \\
\lambda_{1}=\frac{2\left[k\left(2 b c-c^{2}\right)+b q(p-1)-1\right]}{n q(1-p) b^{2} t_{1}} \\
\lambda_{2}=\frac{2\left[k\left(2 b c-c^{2}\right)+b q(p-1)-1\right]}{h b^{2} t_{1}^{2}}
\end{array}\right.
$$

Equations (11) and (12) are unified and expressed as

$$
a_{F}=\lambda_{0}^{\prime}\left(L-X_{n}\right)+\lambda_{1}^{\prime}\left(v_{L}-v_{F}\right)+\lambda_{2}^{\prime}\left(L-L_{3}\right)
$$

where $\lambda_{0}^{\prime}, \lambda_{1}^{\prime}, \lambda_{2}^{\prime}$ are the undetermined parameters, obtained by experiment; $L$ is the instantaneous relative distance between the vehicle pair; $v_{L}$ and $v_{F}$ are the instantaneous speeds of the leader and follower, respectively; $X_{n}$ is the required safe distance at the instantaneous speed of the follower; $L_{3}$ is the space headway during secondary balance.

2.2. Parameter Calibration. In order to get the speed and acceleration data from the camera, Wu et al. [19] and Zhu et al. [20] used the video analysis software called "TrajCap," which can accurately identify the vehicle model and the distribution position of the wheel track in the lane and transform the coordinates in the video to the actual road coordinates. The vehicle trajectory data of the eastbound I-80 interstate highway in Emeryville from the Federal Highway Administration NGSIM data set were used in this paper. The data were collected by seven cameras installed on the 30-storey Pacific Park Plaza building on Christie Avenue at 10 frames per second. The study area is 503-meter long and is divided into 6 lanes. Lane 1 is a High Occupancy Vehicle Lanes (HOV), and lane 6 is a collector-distributor lane, as shown in Figure 4.

In order to ensure the universality of vehicle-following behavior, vehicle trajectory data from $2: 30$ p.m. to $4: 45$ p.m. were selected for research. In order to avoid the potential difference of car-following behavior between different types of vehicles, we processed the existing data sets, that is, extracted the vehicle data set with the vehiclefollowing relationship, only studied the vehicle-following behavior between compact vehicles, and removed the vehicle data in the HOV lane and the collector-distributor lane to ensure that the vehicles under study have similar driving behaviors. We used single-lane data to avoid the impact of lane changing behavior on vehicle-following behavior. A total of 2880 sets of data were obtained after data processing.

Statistical data shows that there is a strong correlation between vehicle-following speed and space headway. As shown in Figure 5, the faster the speed, the larger the space headway. But there is also a phenomenon that follower's speed is very fast and keep a close headway with the leader. In addition, following speed's distribution is most concentrated when the space headway is between $60 \mathrm{~m}$ and $80 \mathrm{~m}$, while following speed's distribution is most dispersed when the space headway is less than $20 \mathrm{~m}$. And the vehiclefollowing speed of all groups shows a normal symmetrical distribution. It should be noted that the parameters of the model are calibrated according to the statistical data range in Figure 5.

The required safe distance of the vehicle at different following speeds is related to the driver's reaction time and the braking performance of the vehicle. The relational expression can be expressed by the following equation:

$$
X_{n}=L_{s}+L_{r}
$$

where $L_{s}$ is the braking distance, and it can be calculated from $L_{s}=v^{2} / 2 \mu g$. Under normal conditions, the friction coefficient $\mu$ of asphalt road is taken as $0.6 ; L_{r}$ is the vehicle's driving distance during the reaction time.

Due to the differences in the driver's reaction time, in order to make the model more accurate, it is necessary to determine the driver's reaction time reasonably. Zhang 


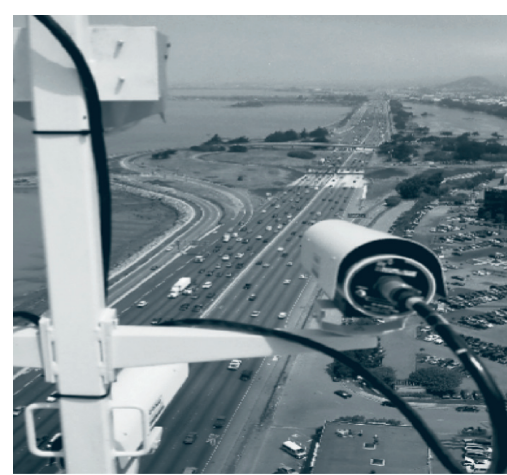

(a)

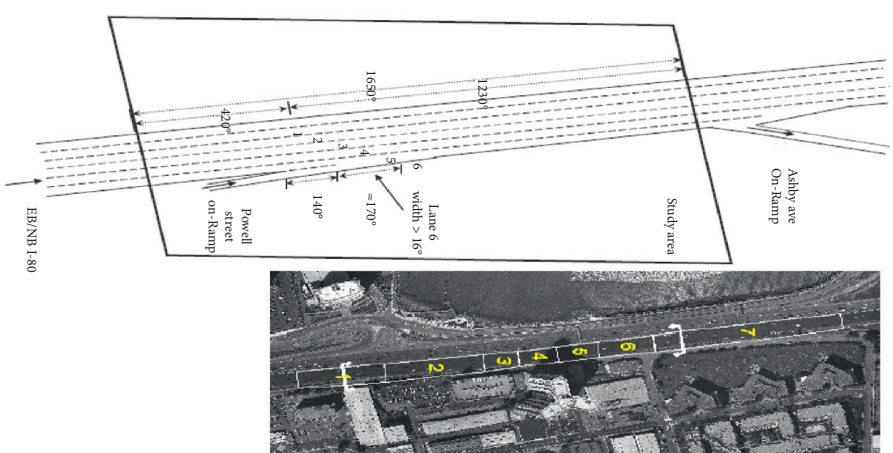

(b)

Figure 4: Summary of data acquisition area.

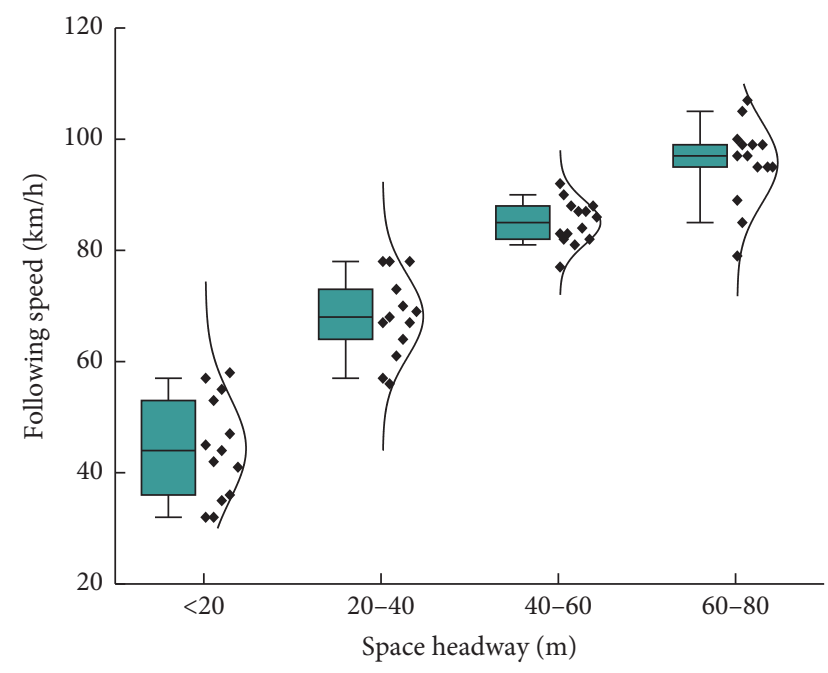

FIgURE 5: Following speed under different following distance.

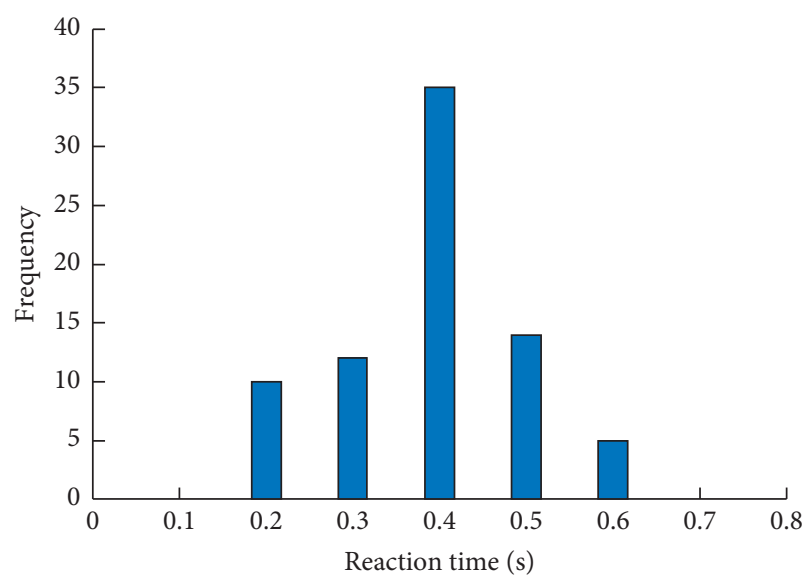

Figure 6: Reaction time histogram.

and Bham [21] studied the vehicle trajectory to obtain the driver's reaction time distribution of following vehicle, as shown in Figure 6. Through calculation, the mean value of the reaction time is $0.396 \mathrm{~s}$, and the standard deviation is 0.109. In this paper, $0.4 \pm 0.1 \mathrm{~s}$ is taken as the driver's reaction time of the following vehicle. After calculation,

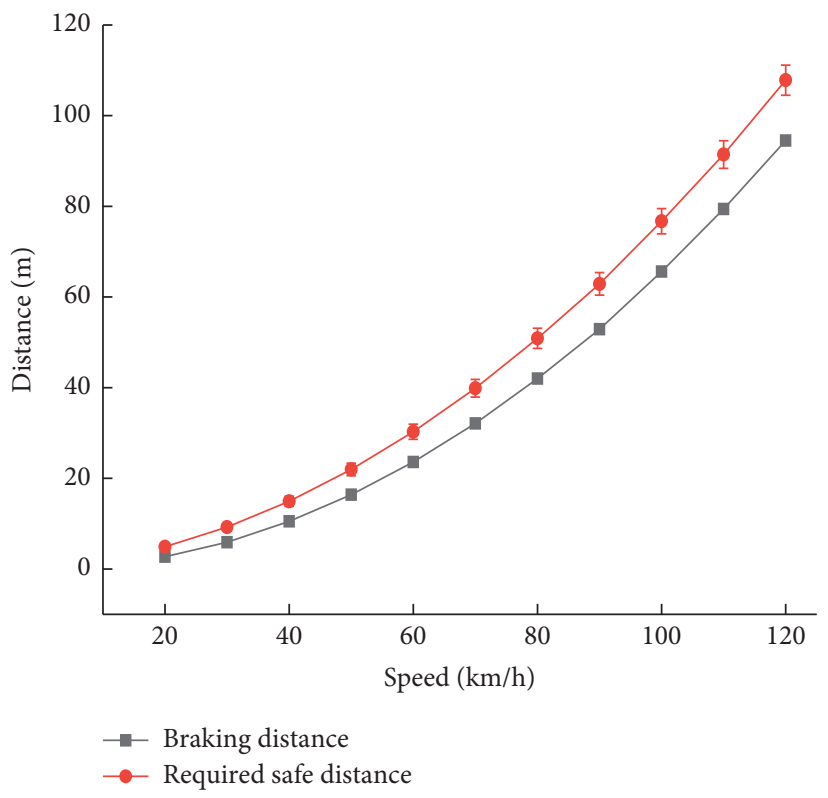

FIGURE 7: Corresponding relationship between vehicle speed's and required safe distance.

TABle 1: Parameter calibration results of molecular following model.

\begin{tabular}{lcccc}
\hline Vehicle-following state & Samples & $\lambda_{0}$ & $\lambda_{1}$ & $\lambda_{2}$ \\
\hline Acceleration & 102 & 3.214 & 0.421 & 0.863 \\
Deceleration & 117 & 2.765 & 0.464 & -3.159 \\
\hline
\end{tabular}

the correspondence between the vehicle's speed and the required safe distance can be obtained, as shown in Figure 7.

Since the model's parameters corresponding to different vehicle's running states are different, we select 50 groups of vehicle-following data in each vehicle-following state and use the genetic algorithm described in [22] to solve them. The results of the model's parameters are shown in Table 1.

After calibrating the parameters of the model, we can simulate and verify the algorithm through the block diagram in Figure 8. 


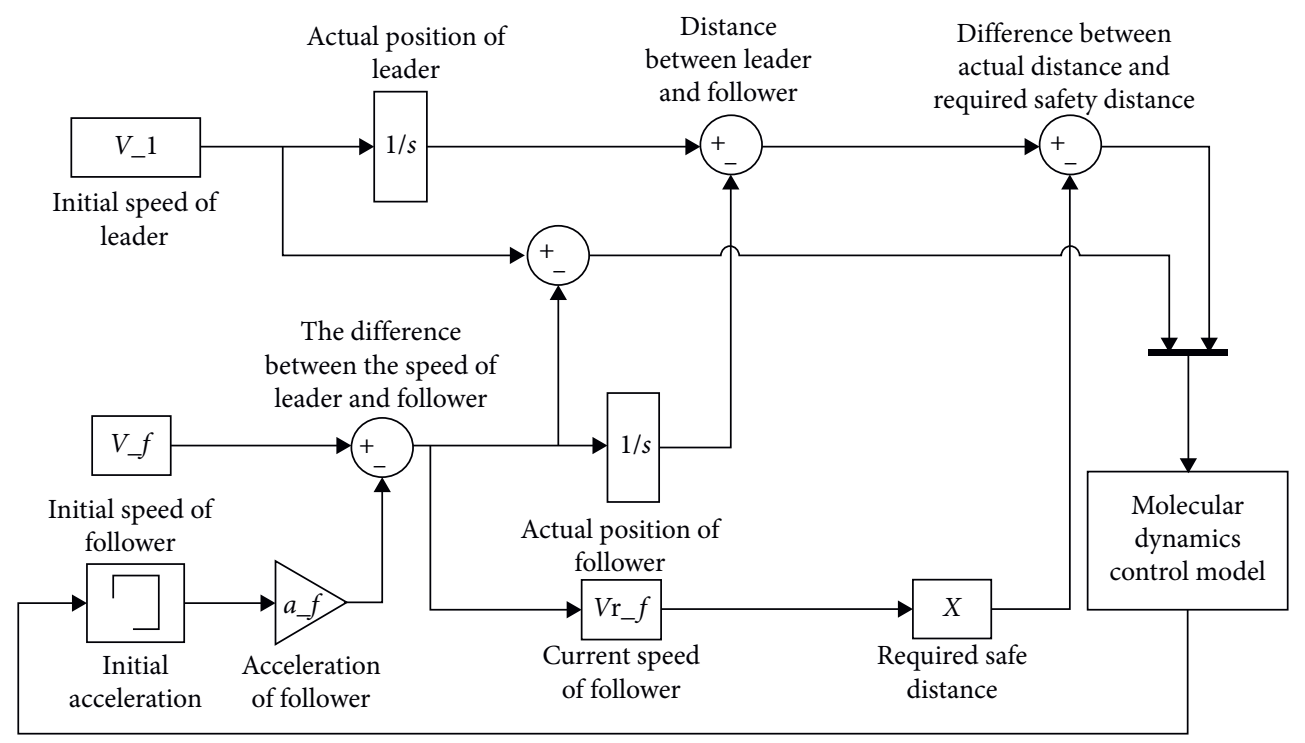

FIGURE 8: Block diagram of the simulation process.

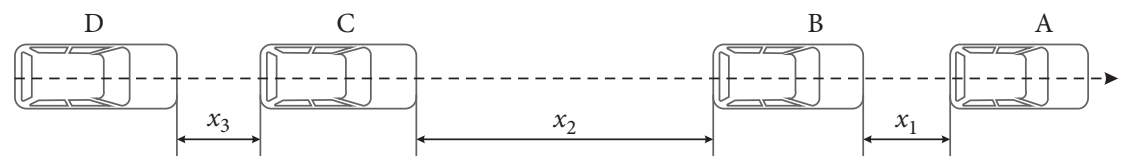

FIgURE 9: Initial relative position of single-lane vehicle following.

2.3. Numerical Simulation of the Model. In order to verify the rationality of the molecular vehicle-following model, we choose to compare it with the classic OV model. After decades of development, the OV model has produced many forms, among which the model proposed by Bando is typical [23], and the model expression is

$$
a(t)=\alpha\left\{V_{\mathrm{ov}}-V(t)\right\}
$$

where $a(t)$ is the acceleration of the follower; $t$ is time; $\alpha$ is the sensitivity coefficient; $V_{\text {ov }}$ is the optimal velocity function.

We choose $V_{\text {ov }}=V_{1}+V_{2} \cdot \tan h\left[C_{1} \cdot\left(x_{\text {gap }}-l_{c}\right)-C_{2}\right]$ as the optimal velocity function, where $C_{1}$ and $C_{2}$ are dimensionless undetermined parameters; we use the same data set to calibrate the $\mathrm{OV}$ model parameters; $\alpha$ is 0.52 ; $C_{1}$ and $C_{2}$ are 0.15 and 1.7 , respectively; $l_{c}$ is the effective size of leader, which is taken as $5 \mathrm{~m}$. After calculation, we can obtain $V_{1}=6.72 \mathrm{~m} / \mathrm{s}$ and $V_{2}=7.86 \mathrm{~m} / \mathrm{s}$.

In the simulation, the friction coefficient of the asphalt pavement is 0.6 , and the number of vehicles is 4 . We assume that the dynamic performance of each vehicle is the same, and they are numbered A to D in the order of the front and rear of the vehicle, as shown in Figure 9. The trajectory of the first vehicle A under the two following models is input based on the measured data, and we get the spatiotemporal trajectory of the vehicles during the period from $25 \mathrm{~s}$ to $115 \mathrm{~s}$, as shown in Figure 10.

\section{Results and Discussion}

Figures 10(a) and 10(b) show the process of the location change caused by the change of the leading vehicle's speed under different models. It can be seen that there are differences in the reactions of the follower to the leader in different running states. Specifically, as can be seen from Figure 11, for the leader A and the follower B with close headway, the deceleration of $\mathrm{B}$ under molecular vehiclefollowing model is more sensitive than that of OV model in the initial 30 40 s deceleration phase. That is to say, the follower B decelerates at higher acceleration. For the leader B and follower $\mathrm{C}$ with long distance, the deceleration change of $\mathrm{B}$ has less stimulation on $\mathrm{C}$ in the molecular following model than in the OV model; that is, the follower $\mathrm{C}$ decelerates with a relatively small acceleration.

During the acceleration period between $50 \mathrm{~s}$ and $70 \mathrm{~s}$ in Figure 11, the speed change of vehicle B in the molecular vehicle-following model is more gentle than that in the $\mathrm{OV}$ following model; it is shown that the vehicle B accelerates and follows with a relatively small acceleration. Comparing the two states of acceleration and deceleration, in some words, the acceleration process lasts for a long time, and the absolute value of acceleration is small as a whole; the duration of vehicle deceleration process is short, and the absolute value of acceleration is large as a whole. That is to say, the acceleration process of the vehicle is slower and the deceleration process is more rapid, and the degree of 


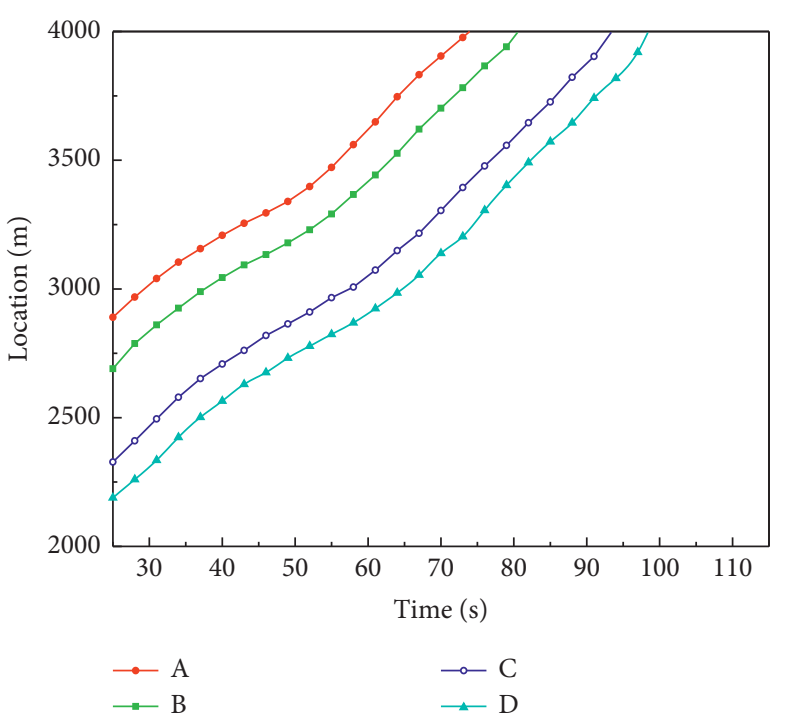

(a)

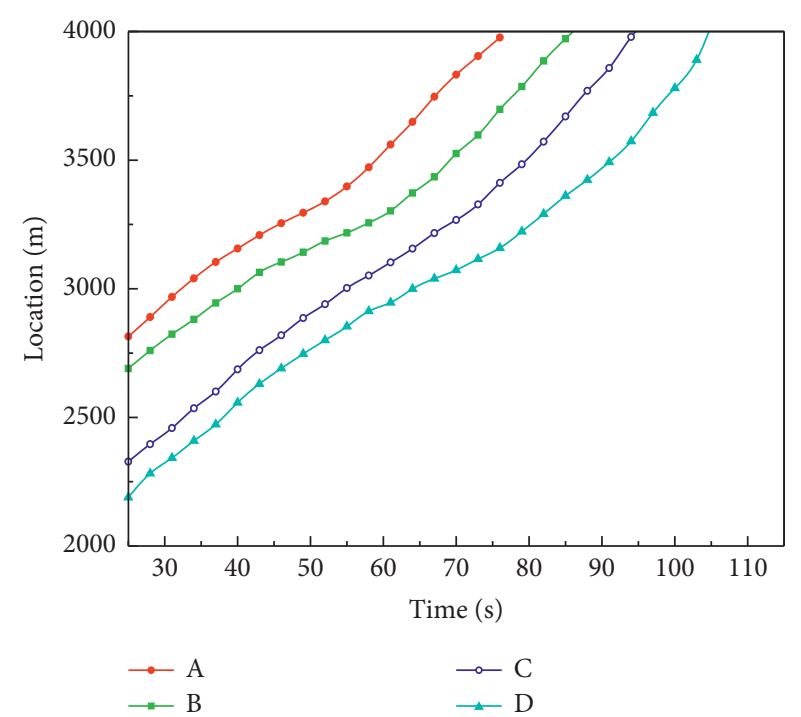

(b)

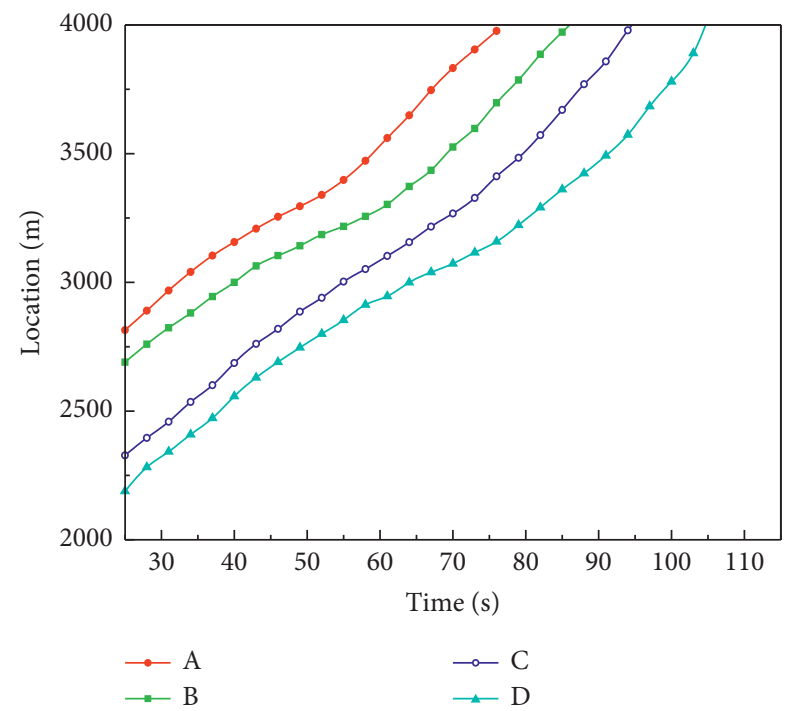

(c)

FIgURE 10: Comparison of spatiotemporal trajectories. (a) OV following model. (b) Molecular following model. (c) Measured data.

reaction to long-distance following operations is weaker than that at close range, which reflects the asymmetry of the car-following process, which is very consistent with the theoretical analysis and the actual situation.

Under the guidance of the same measured vehicle A, taking the measured speed data of measured vehicles B, C, and $\mathrm{D}$ as the benchmark (set as 0 ), the error between the simulated speed and the measured speed of the same vehicle under different models is analyzed to compare the reliability of the model in Figure 12. For vehicle B which keeps close following with vehicle $A$, the absolute value of the error between the simulated speed and the measured speed fluctuates in the range of $[0,2.48]$. The maximum speed error of MF model is 0.87 , while that of OV model is 2.48 . It can also be seen that, under the MF model, the error of the simulated car is closer to the baseline in the whole process, indicating that it is closer to the real value.

For vehicle $\mathrm{C}$ which keeps long distance following with vehicle $B$, the speed error generated under the two models will increase correspondingly. The absolute value of the speed error fluctuates in the range of $[0,4.92]$, while for vehicle $\mathrm{D}$ which keeps following with vehicle $\mathrm{C}$ at close distance, the absolute value of its speed error is up to 6.10 under the OV model and 3.27 under the MF model. The reason may be that vehicle $\mathrm{C}$, which is following vehicle $\mathrm{B}$ at a long distance, has accumulated a large speed error during the driving process, which causes the error to propagate backward and causes the error of the vehicle $\mathrm{D}$ to increase. But whether it is a vehicle $C$ or vehicle $D$, from the perspective of the whole process, its error is still closer to the 


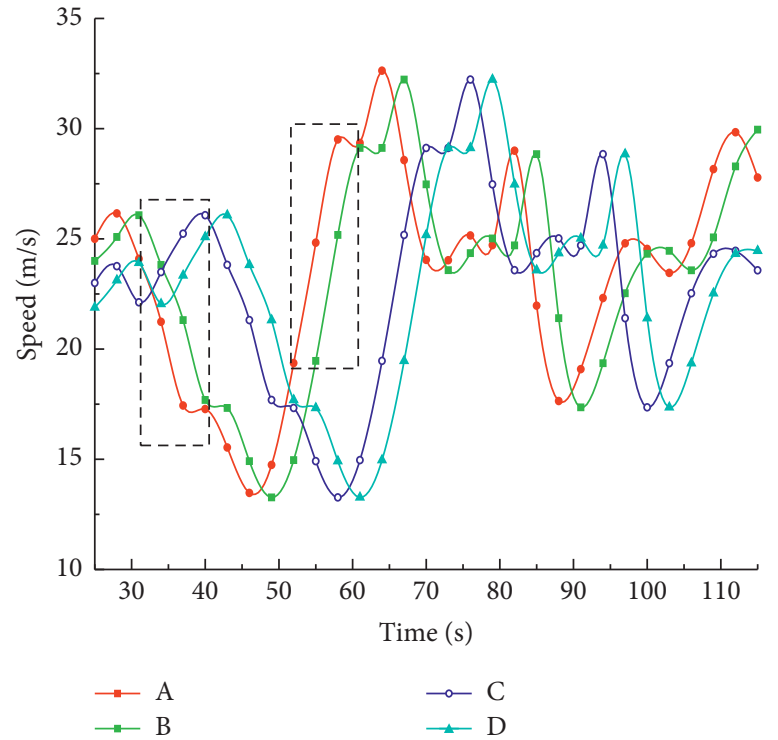

(a)

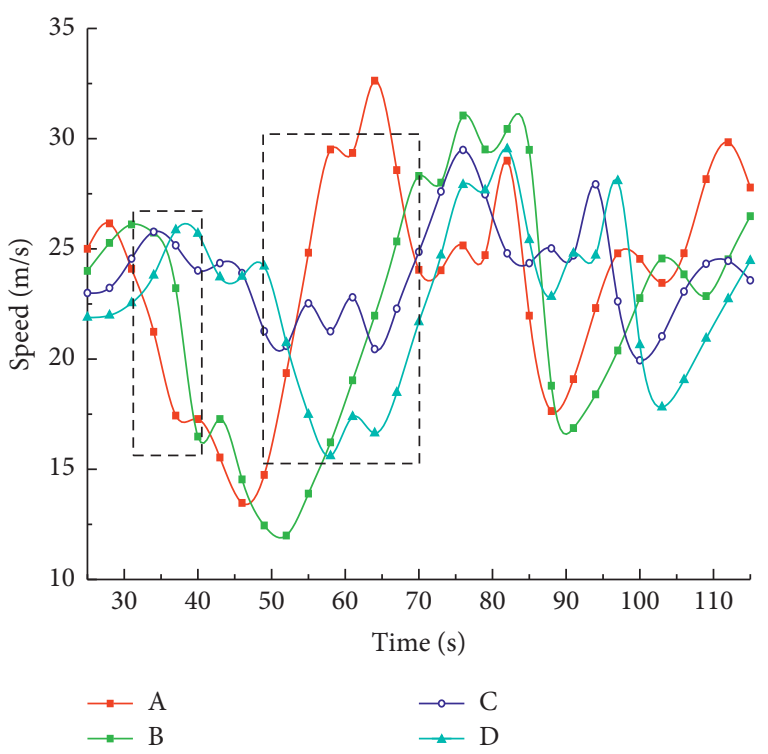

(b)

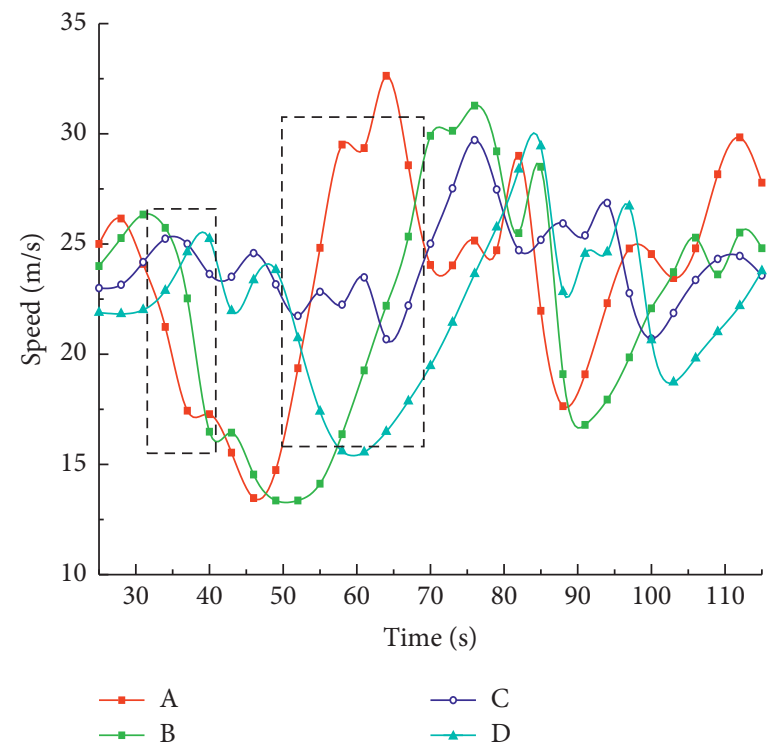

(c)

Figure 11: Measured speed curve and speed curves under different vehicle-following models. (a) OV following model. (b) Molecular following model. (c) Measured data.

baseline under the MF model, which proves that its speed is closer to the true value.

To further verify the accuracy of the MF model, the mean absolute error (MAE) and root mean square error (RMSE) are used as the model evaluation indicators, and the mathematical expression is

$$
\begin{aligned}
& M_{\mathrm{MAE}}=\frac{1}{N} \sum_{i=1}^{N}\left|y_{i}-\hat{y}_{i}\right|, \\
& R_{\mathrm{RMSE}}=\sqrt{\frac{1}{N} \sum_{i=1}^{N}\left(y_{i}-\hat{y}_{i}\right)^{2},}
\end{aligned}
$$

where $y_{i}$ represents the actual data; $\hat{y}_{i}$ represents the data obtained by the model.

It can be seen from the evaluation results in Table 2 that the mean values of $R_{\mathrm{RMSE}}$ and $M_{\mathrm{MAE}}$ in the molecular vehicle-following model are less than the OV following model, regardless of whether the vehicle is accelerating or decelerating. It proves that the model built can more accurately reflect the vehicle-following behavior. The essence can be discussed from the perspective of molecular physical mechanics: (1) When the adjacent vehicle's distance is gradually smaller than the required safe distance, that is, $x_{1}<X_{n}^{1}$, the two vehicles behave as repulsion. The repulsive force received increases with the continuous decrease of the distance, and the driver of following vehicle adopts a more 


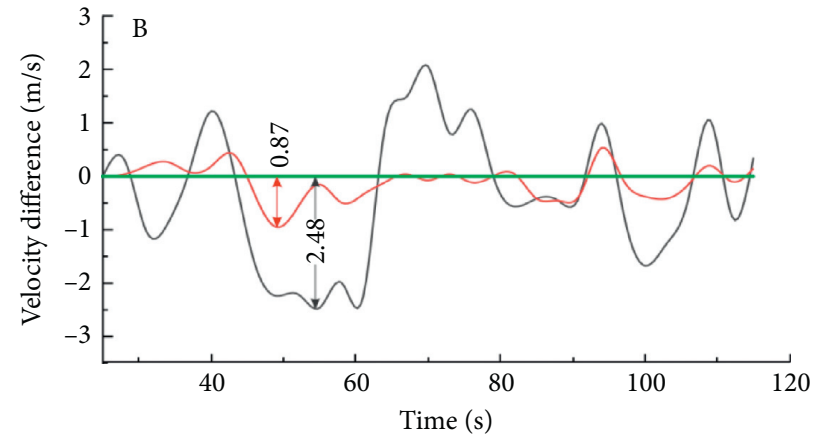

$\mathrm{OV}$
$\mathrm{MF}$

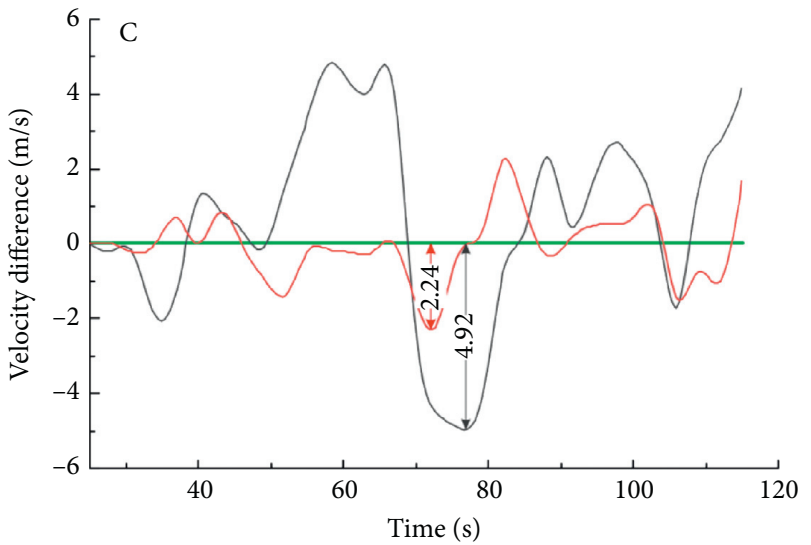

- OV

- MF

(b)

(a)

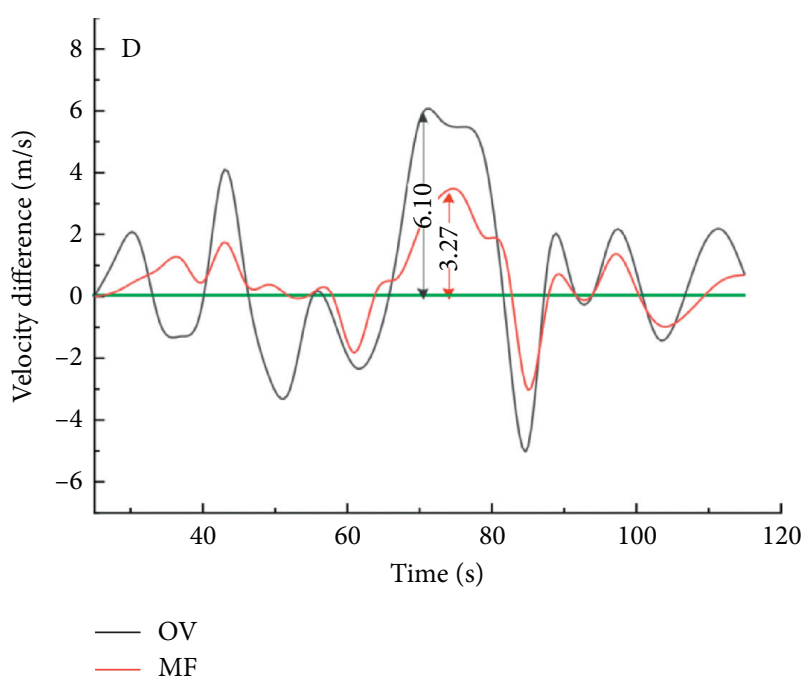

(c)

FIgURE 12: Velocity error curves of vehicles B, C, and D.

TABLE 2: Different model evaluation results.

\begin{tabular}{lccc}
\hline \multirow{2}{*}{ Vehicle-following state } & Model & \multicolumn{2}{c}{ Evaluation } \\
& & $M_{\mathrm{MAE}}$ & $R_{\mathrm{RMSE}}$ \\
\hline Accelerate & OV following & 0.314 & 12.577 \\
Accelerate & Molecular following & 0.175 & 7.346 \\
Decelerate & OV following & 0.288 & 15.331 \\
Decelerate & Molecular following & 0.142 & 9.215 \\
\hline
\end{tabular}

aggressive deceleration operation so that the vehicle decelerates with gradually increasing acceleration. (2) For close-distance vehicles, when the distance between vehicle pair is gradually farther than the required safe distance, that is, $x_{2}>X_{\mathrm{n}}^{2}$, two vehicles behave as gravitation. The gravitational force suffered decreases with the continuous increase of the vehicle distance in the early stage, and the driver accelerates in a relatively gentle manner. That also confirms that the driver is more inclined to choose an acceptable and comfortable way to accelerate, rather than choosing the optimal solution. (3) For long-distance vehicles, that is, $x_{3} \gg X_{n}^{3}$, the follower accelerates slightly by the small gravitational force, and the corresponding reality is that the vehicle running at a high speed has less accelerating space and the accelerating requirement is also smaller.

\section{Conclusion}

In this paper, the molecular vehicle-following model established from the perspective of molecular mechanics can better explain the driver's asymmetry between acceleration and deceleration in the vehicle-following process at different distances and makes up for the defect that the traditional vehicle-following model ignores the driver's psychological characteristics. The numerical simulation results show that 
the acceleration of vehicle during acceleration and deceleration is not fixed under the molecular vehicle-following model. Precisely speaking, following vehicle is more sensitive to the deceleration of the leading vehicle than acceleration, and the following vehicle is more sensitive to the acceleration/deceleration of the leading vehicle in a short distance than that of the leading vehicle in a long distance. The results of the simulation can be well analyzed from the intermolecular forces. The built model can better describe the driver's following behavior, so that following vehicle can better predict the speed fluctuation of the leading vehicle. The next step is to apply the model to the following behavior of the connected autonomous vehicle. And we plan to study the comfort and safety of following vehicles under the molecular following model by calculating the jerk and setting the threshold of force.

\section{Data Availability}

The original codes of the numerical tests used to support the findings of this study are available from the corresponding author upon request.

\section{Conflicts of Interest}

The authors declare that there are no conflicts of interest regarding the publication of this paper.

\section{Authors' Contributions}

Dayi Qu and Yanfeng Jia conceived and designed the research. Xiaolong Ma performed the experiments. Lu Lin and Jiale Hong analyzed the data. Yanfeng Jia wrote the paper. All authors read and approved the final paper.

\section{Acknowledgments}

This project was supported by the National Natural Science Foundation of China (Grant no. 51678320).

\section{References}

[1] L. A. Pipes, "An operational analysis of traffic dynamics," Journal of Applied Physics, vol. 24, no. 3, pp. 274-281, 1953.

[2] D. E. Rumelhart, G. E. Hinton, and R. J. Williams, "Learning representations by back-propagating errors," Nature, vol. 323, no. 6088, pp. 533-536, 1986.

[3] N. Kehtarnavaz, N. Groswold, K. Miller, and P. Lascoe, "A transportable neural-network approach to autonomous vehicle following," IEEE Transactions on Vehicular Technology, vol. 47, no. 2, pp. 694-702, 1998.

[4] H. F. Jia, Z. C. Jun, and X. Y. Wang, "Development of a carfollowing model based on artificial neural networks," Journal of Highway and Transportation Research and Development, vol. 18, no. 4, pp. 92-94, 2001.

[5] D. D. Ding, L. Sun, and S. Chen, "Machine learning-dynamics coupled vehicle following model," Journal of Transportation Systems Engineering and Information Technology, vol. 17, no. 06, pp. 37-43, 2017.

[6] Q. Sun and Z. Y. Guo, "Vehicle following model based on LSTM neural network," Journal of Jilin University, vol. 50, no. 4, pp. 1380-1386, 2020.
[7] S. Kikuchi and P. Chakroborty, "Car-following model based on fuzzy inference system," Journal of the Transportation Research Board, vol. 1365, pp. 82-91, 1992.

[8] X. Ma, "A neural-fuzzy framework for modeling car-following behavior," in Proceedings of the IEEE International Conference on Systems, Man and Cybernetics, pp. 1178-1183, Taipei, Taiwan, October 2006.

[9] D. Wei and H. Liu, "Analysis of asymmetric driving behavior using a self-learning approach," Transportation Research Part B-Methodological, vol. 47, pp. 1-14, 2013.

[10] H. P. Wang and F. Chen, "Research on vehicle following model based on online support vector regression," Electronic Technology, vol. 47, no. 11, pp. 21-28, 2018.

[11] D. C. Gazis, R. Herman, R. W. Rothery et al., "Nonlinear follow-the-leader models of traffic flow," Operations Research, vol. 9, no. 4, pp. 545-567, 1961.

[12] G. F. Newell, "Nonlinear effects in the dynamics of car following," Operations Research, vol. 9, pp. 209-229, 1961.

[13] D. X. Xia and C. G. Li, "Hydrodynamic model for two-lane traffic flow considering the information of multi-vehicles ahead and lateral vehicles," Systems Engineering-Theory \& Practice, vol. 32, no. 07, pp. 1562-1567, 2012.

[14] D. H. Sun, Y. F. Li, and C. Tian, "Car-following model based on the information of multiple ahead \& velocity difference," Systems Engineering-Theory \& Practice, vol. 30, no. 7, pp. 1326-1332, 2010.

[15] C. Zhai, W. T. Wu, W. M. Liu et al., "Modeling and simulation of traffic flow dynamics based on cognitive differences," Journal of Transportation Systems Engineering and Information Technology, vol. 18, no. 06, pp. 148-156, 2018.

[16] M. Zhu, X. Wang, A. Tarko, and S. e. Fang, "Modeling carfollowing behavior on urban expressways in Shanghai: a naturalistic driving study," Transportation Research Part C: Emerging Technologies, vol. 93, pp. 425-445, 2018.

[17] H. X. Ge, Y. Cui, and R. J. Cheng, "A car-following model with considering control signals from front and rear," Acta Physica Sinica, vol. 63, no. 11, Article ID 110504, 2014.

[18] X. Wang, R. Jiang, L. Li, Y. Lin, X. Zheng, and F.-Y. Wang, "Capturing car-following behaviors by deep learning," IEEE Transactions on Intelligent Transportation Systems, vol. 19, no. 3, pp. 910-920, 2018.

[19] G. Wu, F. Chen, X. Pan, M. Xu, and X. Zhu, "Using the visual intervention influence of pavement markings for rutting mitigation-part I: preliminary experiments and field tests," International Journal of Pavement Engineering, vol. 20, no. 6, pp. 734-746, 2019.

[20] X. Zhu, Z. Dai, F. Chen, X. Pan, and M. Xu, "Using the visual intervention influence of pavement marking for rutting mitigation-part II: visual intervention timing based on the finite element simulation," International Journal of Pavement Engineering, vol. 20, no. 5, pp. 573-584, 2019.

[21] X. Zhang and G. H. Bham, "Estimation of driver reaction time from detailed vehicle trajectory data," Moas, vol. 7, pp. 574-579, 2007.

[22] Y. Z. Luo, D. C. Yuan, and G. J. Tang, "Hybrid genetic algorithm for solving nonlinear equations," Chinese Journal of Computational Mechanics, vol. 22, no. 1, pp. 109-114, 2005.

[23] M. Bando, K. Hasebe, A. Nakayama, A. Shibata, and Y. Sugiyama, "Dynamical model of traffic congestion and numerical simulation," Physical Review E, vol. 51, no. 2, pp. 1035-1042, 1995. 